\title{
Carbono do solo e atributos de fertilidade em resposta à calagem superficial em plantio direto
}

\author{
Clever Briedis ${ }^{(1)}$, João Carlos de Moraes Sá(1), Eduardo Fávero Caires(1), Jaqueline de Fátima Navarro(1), \\ Thiago Massao Inagaki ${ }^{(1)}$ e Ademir de Oliveira Ferreira ${ }^{(2)}$
}

\begin{abstract}
(1)Universidade Estadual de Ponta Grossa, Departamento de Ciência do Solo e Engenharia Agrícola, Campus de Uvaranas, Avenida Carlos Cavalcanti, № 4.748, CEP 84030-900 Ponta Grossa, PR. E-mail: cleverbriedis@yahoo.com.br, jcmsa@uepg.br, efcaires@uepg.br, jaquenavarro@hotmail.com, thiago811@yahoo.com.br (2)Universidade Federal de Santa Maria, Prédio 42, Laboratório de Manejo e Conservação do Solo e da Água, Avenida Roraima, Camobi, CEP 97105-900 Santa Maria, RS. E-mail: aoferreira1@yahoo.com.br
\end{abstract}

Resumo - O objetivo deste trabalho foi avaliar o efeito da aplicação de calagem superficial sobre o conteúdo de carbono orgânico total (COT) do solo, em plantio direto, e identificar a relação entre esse conteúdo e outros atributos de fertilidade. O experimento foi realizado em Latossolo Vermelho de textura média, em Ponta Grossa, PR. Os tratamentos consistiram da aplicação de calcário dolomítico na superfície do solo, nas doses 0 e $6 \mathrm{Mg} \mathrm{ha}^{-1}$, em 1993, e da reaplicação de 0 e $3 \mathrm{Mg} \mathrm{ha}^{-1}$, em 2000, nas parcelas com e sem calcário. O solo foi coletado em 2008, e foram analisados os conteúdos de COT e os atributos de fertilidade. A calagem produziu aumento do conteúdo de COT e da saturação por bases, e diminuição da saturação por alumínio. O conteúdo de COT apresentou relação linear com a capacidade de troca catiônica (CTC) efetiva e correlação com os teores de P e K. A CTC potencial apresentou correlação com o COT, e a CTC efetiva com o pH. A calagem superficial em sistema plantio direto, em longo período, proporciona aumento no conteúdo de COT e de $\mathrm{N}$ total.

Termos para indexação: atributos químicos, CTC, matéria orgânica do solo, plantio direto.

\section{Soil carbon and fertility attributes in response to surface liming under no-tillage}

\begin{abstract}
The objective of this work was to evaluate the effect of surface liming on the content of total organic carbon (TOC) of a soil under no-tillage, and to identify the relationship between that content and other fertility attributes. The experiment was carried out on a medium-textured Oxisol in Ponta Grossa, PR, Brazil. The treatments consisted of the application of 0 and $6 \mathrm{Mg} \mathrm{ha}^{-1}$ of dolomitic lime on soil surface in 1993 and of the reapplication of 0 and $3 \mathrm{Mg} \mathrm{ha}^{-1}$ in 2000, on plots with or without lime. The soil was collected in 2008, and the TOC content and fertility attributes were evaluated. Liming increased TOC content and base saturation, and decreased aluminium saturation. TOC content showed a linear relation with the effective cation exchange capacity and correlation with the levels of $\mathrm{P}$ and $\mathrm{K}$. The potential CEC showed correlation with TOC, and the effective CEC with $\mathrm{pH}$. Liming under no-tillage, for a long period of time, increases TOC and total $\mathrm{N}$ contents.
\end{abstract}

Index terms: chemical attributes, $\mathrm{CEC}$, soil organic matter, no-tillage.

\section{Introdução}

Os solos em regiões de clima tropical e subtropical, em sua maioria, apresentam predominantemente argilas cauliníticas e óxidos de ferro e alumínio em sua fração argila. Esses colóides geram cargas dependentes de $\mathrm{pH}$ (Soares et al., 2005; Weber et al., 2005) e têm elevado ponto de carga zero (PCZ), em razão do número baixo ou inexistente de cargas negativas permanentes e da alta atratividade de seus grupos funcionais pelo hidrogênio.

A matéria orgânica do solo (MOS) também apresenta carga variável. Contudo, o pH para a dissociação de seus grupos funcionais, principalmente o carboxílico, é muito baixo (Sposito, 2008). Por esse motivo, a MOS, em solos tropicais e subtropicais, é responsável por 75 a $90 \%$ da capacidade de troca catiônica (CTC) do solo (Fontana et al., 2006; Bortoluzzi et al., 2009). Como a MOS tem papel fundamental na determinação da carga líquida negativa dos solos e, portanto, na retenção de nutrientes para as plantas, práticas agrícolas que mantenham ou elevem os teores de carbono orgânico total (COT) no solo são necessárias. Além disso, os solos nessas regiões são geralmente ácidos e apresentam baixas reservas de nutrientes. A calagem é a prática mais eficiente para resolver os problemas relacionados à acidez, à elevação do $\mathrm{pH}$, aos suprimento de $\mathrm{Ca} \mathrm{e}$ $\mathrm{Mg}$, e à saturação por bases (Ernani et al., 2004; Caires et al., 2008; Moreira \& Fageria, 2010). 
Em sistema plantio direto (SPD), a calagem é realizada em superfície sem que ocorra o revolvimento do solo, o que promove a manutenção de agregados e evita a exposição da MOS (Caires et al., 2006a). Contudo, ao elevar o $\mathrm{pH}$ do solo, a calagem também eleva sua atividade microbiana (Ekenler \& Tabatabai, 2003; Mijangos et al., 2010), o que, em curto prazo, pode promover a decomposição mais acelerada da MOS (Yao et al., 2009). Em longo prazo, no entanto, a dinâmica da MOS em relação à calagem pode ser diferente, em razão dos efeitos indiretos relacionados à maior produção de fitomassa por área, atribuídos a melhorias nas condições edáficas e, principalmente, em razão da neutralização do $\mathrm{Al}^{3+}$ (Ridley et al., 1990; Hati et al., 2008).

O objetivo deste trabalho foi avaliar o efeito da aplicação de calagem superficial, sobre o conteúdo de COT no solo, em plantio direto, e identificar a relação entre esse conteúdo e outros atributos de fertilidade.

\section{Material e Métodos}

O experimento foi realizado em Ponta Grossa, PR $\left(25^{\circ} 10^{\prime} \mathrm{S}, 50^{\circ} 5^{\prime} \mathrm{W}\right)$, em um Latossolo Vermelho distrófico de textura média (Santos et al., 2006), com 295, 240 e $465 \mathrm{~g} \mathrm{~kg}^{-1}$ de argila, silte e areia, respectivamente, conforme método de Bouyoucos (Claessen, 1997). Na fração argila, o solo apresentava $90,1 \%$ de caulinita $\left(265,8 \mathrm{~g} \mathrm{~kg}^{-1}\right), 9,1 \%$ de goetita $\left(26,8 \mathrm{~g} \mathrm{~kg}^{-1}\right)$ e $0,8 \%$ de hematita $\left(2,4 \mathrm{~g} \mathrm{~kg}^{-1}\right)$. Antes da instalação do experimento, em maio de 1993, as análises químicas e granulométricas do solo, da camada de 0-20 cm, indicaram: $\mathrm{pH}$ em $\mathrm{CaCl}_{2}$ de 4,5; $6 \mathrm{mmol}_{\mathrm{c}} \mathrm{dm}^{-3}$ de $\mathrm{Al}^{+3} ; 16 \mathrm{mmol}_{\mathrm{c}} \mathrm{dm}^{-3} \mathrm{de} \mathrm{Ca}^{+2}$; $10 \mathrm{mmol}_{\mathrm{c}} \mathrm{dm}^{-3} \mathrm{de}_{\mathrm{Mg}^{+2}} ; 1,4 \mathrm{mmol}_{\mathrm{c}} \mathrm{dm}^{-3}$ de $\mathrm{K}^{+}$; acidez potencial $(\mathrm{H}+\mathrm{Al})$ de $58 \mathrm{mmol}_{\mathrm{c}} \mathrm{dm}^{-3} ; 9 \mathrm{mg} \mathrm{dm}^{-3} \mathrm{de}$ P; $19 \mathrm{~g} \mathrm{dm}^{-3}$ de COT; e saturação por bases de $32 \%$ (Pavan et al., 1992).

O clima da região, conforme a classificação de Köppen, é do tipo $\mathrm{Cfb}$, subtropical úmido mesotérmico, com verões frescos e ocorrência de geadas severas e frequentes no inverno, sem estação seca definida. A temperatura média anual é de $17,8^{\circ} \mathrm{C}$, e a precipitação pluvial média anual de $1.553 \mathrm{~mm}$. Até a instalação do experimento, a área vinha sendo cultivada no SPD por 15 anos: em 1969, com conversão da vegetação em áreas de lavoura; de 1969 a 1978, com três cultivos de arroz e sete cultivos com sucessão soja e trigo, sob plantio convencional; de 1978 a 1993, com 15 anos com sequência de cultivos sob SPD; e de 1993 a 2008, período do experimento com calagem sob SPD.

Utilizou-se o delineamento experimental de blocos ao acaso, com três repetições. As parcelas, cujas dimensões foram de 4,0x6,3 m, consistiram da aplicação de calcário dolomítico na superfície do solo, na dose de 0 e $6 \mathrm{Mg} \mathrm{ha}^{-1}$, em 1993. Em 2000, realizou-se a reaplicação de calcário na dose de 0 e $3 \mathrm{Mg} \mathrm{ha}^{-1}$ nas parcelas que haviam ou não recebido tratamento prévio com calcário em 1993. Os seguintes tratamentos foram formados: $0+0\left(0 \mathrm{Mg} \mathrm{ha}^{-1} \mathrm{em} 1993\right.$ e $0 \mathrm{Mg} \mathrm{ha}^{-1}$ em 2000, que representou o controle); $6+0$ (6 $\mathrm{Mg} \mathrm{ha}^{-1}$ em 1993 e $0 \mathrm{Mg} \mathrm{ha}^{-1}$ em 2000); 0+3 (0 $\mathrm{Mg} \mathrm{ha}^{-1}$ em 1993 e $3 \mathrm{Mg} \mathrm{ha}^{-1}$ em 2000) e 6+3 (6 $\mathrm{Mg} \mathrm{ha}^{-1} \mathrm{em}$ 1993 e $3 \mathrm{Mg} \mathrm{ha}^{-1}$ em 2000). A dose de $6 \mathrm{Mg} \mathrm{ha}^{-1} \mathrm{em}$ 1993 foi aplicada para elevar a saturação por bases da camada de 0-20 cm do solo para aproximadamente $90 \%$. A reaplicação de $3 \mathrm{Mg} \mathrm{ha}^{-1}$, em 2000, foi calculada com base na análise do solo, com vistas à elevação da saturação por bases para $65 \%$, tendo-se considerado a análise química da amostra retirada da camada de 0-20 cm, no tratamento com $6 \mathrm{Mg} \mathrm{ha}^{-1}$ de calcário em 1993. As subparcelas foram constituídas pelas camadas de coleta de solo, nas profundidades de $0-2,5 ; 2,5-5$; $5-10$ e $10-20 \mathrm{~cm}$.

O calcário dolomítico utilizado em 1993 continha $176 \mathrm{~g} \mathrm{~kg}^{-1}$ de $\mathrm{Ca}, 136 \mathrm{~g} \mathrm{~kg}^{-1}$ de $\mathrm{Mg}$ e poder relativo de neutralização total (PRNT) de $84 \%$, enquanto o usado em 2000 continha $196 \mathrm{~g} \mathrm{~kg}^{-1} \mathrm{de} \mathrm{Ca}, 130 \mathrm{~g} \mathrm{~kg}^{-1} \mathrm{de} \mathrm{Mg}$ e 90\% de PRNT. Durante os 15 anos do experimento, foi mantida a mesma sequência de culturas para todas as parcelas experimentais. A entrada anual de $\mathrm{C}$ oriundo de resíduos culturais (parte aérea e raízes), em cada tratamento, foi estimada com base no índice de colheita (IC) da produção de grãos e na relação raiz/parte aérea. $\mathrm{O}$ conteúdo de $\mathrm{C}$ na matéria seca foi determinado em cada espécie, e utilizado para o cálculo da quantidade de C adicionada (Sá et al., 2001). Os aportes de matéria seca e de $C$ no período avaliado são apresentados na Tabela 1.

As amostras do solo foram coletadas logo após o manejo da cultura de aveia-preta, nas parcelas experimentais, em outubro de 2008, com uso de minitrincheiras com dimensões de $20 \times 20 \mathrm{~cm}$. Após a coleta, as amostras de solo foram secadas ao ar, passadas em peneira de $2 \mathrm{~mm}$ e armazenadas em sacos de plástico. 
O conteúdo de COT e nitrogênio total (NT) foram determinados pelo método da combustão seca, com uso de determinador elementar de C e N TruSpec CN Leco 2006 (Leco Corporation, St. Joseph, MI, EUA).

$\mathrm{O}$ pH do solo foi determinado em suspensão de $\mathrm{CaCl}_{2}$, a $0,01 \mathrm{~mol} \mathrm{~L} \mathrm{~L}^{-1}\left(1: 2,5\right.$ solo/solução; $\left.\mathrm{v} \mathrm{v} \mathrm{v}^{-1}\right)$. $\mathrm{O} \mathrm{Al}^{3+}$ trocável, o $\mathrm{Ca}^{2+}$ e o $\mathrm{Mg}^{2+}$ foram extraídos com $\mathrm{KCl}$ neutro a $1,0 \mathrm{~mol} \mathrm{~L}^{-1}$, e o $\mathrm{K}^{+}$com Mehlich-1, em uma relação solo/solução 1:10 $\left(\mathrm{v} \mathrm{v}^{-1}\right)$. Foram determinados o $\mathrm{Al}^{3+}$ (acidez trocável $-\mathrm{KCl}$ ), por titulação com $\mathrm{NaOH} 0,025 \mathrm{~mol} \mathrm{~L}^{-1}$; o $\mathrm{Ca}^{2+}$ e o $\mathrm{Mg}^{2+}$, por titulação com EDTA 0,025 mol L-1; e o $\mathrm{K}^{+}$, por fotometria de chama (Pavan et al., 1992). A CTC potencial (CTCp) foi calculada a partir da soma das bases trocáveis $\left(\mathrm{Ca}^{2+}, \mathrm{Mg}^{2+}, \mathrm{K}^{+}\right)$mais $\mathrm{H}^{+}+\mathrm{Al}^{3+}$. A CTC efetiva (CTCe) foi calculada a partir da soma de bases mais $\mathrm{Al}^{3+}$. A saturação por bases $(\mathrm{V})$ foi calculada pela fórmula: $\mathrm{V}(\%)=100\left(\mathrm{Ca}^{2+}+\mathrm{Mg}^{2+}+\mathrm{K}^{+}\right) / \mathrm{CTC}$. Já a saturação por alumínio $(\mathrm{m} \%)$, foi calculada pela fórmula: $\mathrm{m}(\%)=100\left(\mathrm{Al}^{3+} / \mathrm{CTCe}\right)$.

Tabela 1. Aportes anuais $\left(\mathrm{Mg} \mathrm{ha}^{-1}\right)$ de resíduos culturais (RC) e carbono (C), em razão da sequência de culturas, referentes ao período de 1994 a 2008, nos tratamentos com doses de calcário em superfície, em Latossolo Vermelho sob sistema plantio direto.

\begin{tabular}{|c|c|c|c|c|c|c|c|c|c|c|}
\hline \multirow[t]{3}{*}{$\overline{\text { Ano }}$} & \multirow{3}{*}{\multicolumn{2}{|c|}{$\frac{\text { Culturas }^{(1)}}{\text { Verão Inverno }}$}} & \multicolumn{8}{|c|}{ Tratamentos $^{(2)}$} \\
\hline & & & \multicolumn{2}{|c|}{$0+0$} & \multicolumn{2}{|c|}{$6+0$} & \multicolumn{2}{|c|}{$0+3$} & \multicolumn{2}{|c|}{$6+3$} \\
\hline & & & $\mathrm{RC}$ & $\mathrm{C}$ & $\mathrm{RC}$ & $\mathrm{C}$ & $\mathrm{RC}$ & $\mathrm{C}$ & $\mathrm{RC}$ & $\mathrm{C}$ \\
\hline 1994 & $\mathrm{Sj}$ & $\mathrm{Er}+\mathrm{Ap}$ & 7,3 & 3,0 & 8,7 & 3,6 & 7,3 & 3,0 & 8,7 & 3,6 \\
\hline 1995 & MI & $\mathrm{P}$ & 14,1 & 6,4 & 17,1 & 7,7 & 14,1 & 6,4 & 17,1 & 7,7 \\
\hline 1996 & $\mathrm{Sj}$ & $\operatorname{Tr}$ & 5,4 & 2,2 & 5,9 & 2,4 & 5,4 & 2,2 & 5,9 & 2,4 \\
\hline 1997 & $\mathrm{Sj}$ & $\mathrm{Tt}$ & 4,9 & 2,0 & 5,2 & 2,2 & 4,9 & 2,0 & 5,2 & 2,2 \\
\hline 1998 & $\mathrm{Sj}$ & Ap & 6,0 & 2,5 & 7,2 & 3,0 & 6,0 & 2,5 & 7,2 & 3,0 \\
\hline 1999 & $\mathrm{Sj}$ & Ap & 6,7 & 2,8 & 8,1 & 3,4 & 6,7 & 2,8 & 8,1 & 3,4 \\
\hline 2000 & $\mathrm{Sj}$ & Ap & 7,9 & 3,2 & 8,7 & 3,6 & 7,7 & 3,2 & 8,9 & 3,7 \\
\hline 2001 & Ml & Ap & 16,8 & 7,5 & 20,6 & 9,2 & 18,5 & 8,3 & 21,2 & 9,5 \\
\hline 2002 & $\mathrm{Sj}$ & Ap & 7,7 & 3,2 & 10,0 & 4,2 & 9,9 & 4,1 & 10,8 & 4,5 \\
\hline 2003 & $\mathrm{Sj}$ & $\operatorname{Tr}$ & 5,8 & 2,4 & 8,7 & 3,7 & 8,0 & 3,4 & 10,7 & 4,6 \\
\hline 2004 & $\mathrm{Sj}$ & Ap & 7,2 & 3,0 & 9,4 & 3,9 & 9,3 & 3,9 & 10,6 & 4,4 \\
\hline 2005 & Ml & Ap & 13,2 & 5,9 & 19,4 & 8,7 & 19,1 & 8,6 & 21,1 & 9,5 \\
\hline 2006 & $\mathrm{Sj}$ & Ap & 7,6 & 3,1 & 10,5 & 4,3 & 9,6 & 4,0 & 11,4 & 4,7 \\
\hline 2007 & $\mathrm{Sj}$ & Ap & 6,9 & 2,9 & 9,1 & 3,8 & 9,2 & 3,9 & 10,7 & 4,5 \\
\hline 2008 & $\mathrm{Sj}$ & Ap & 7,1 & 2,4 & 9,4 & 2,5 & 9,5 & 2,6 & 10,9 & 3,0 \\
\hline Total & - & - & 124,5 & 52,5 & 158,0 & 66,1 & 145,3 & 60,7 & 168,5 & 70,5 \\
\hline
\end{tabular}

${ }^{(1)} \mathrm{Sj}$, soja; Er+Ap, consórcio de ervilhaca e aveia-preta; MI, milho; P, pousio; $\mathrm{Tr}$, trigo; Tt, triticale; Ap, aveia-preta. ${ }^{(2)}$ Doses de calcário: $0+0$ (0 Mg ha-1 em 1993 e $0 \mathrm{Mg} \mathrm{ha}^{-1}$ em 2000), 6+0 (6 Mg ha- em 1993 e $0 \mathrm{Mg} \mathrm{ha}^{-1}$ em 2000), 0+3 (0 Mg ha- em 1993 e $3 \mathrm{Mg} \mathrm{ha}^{-1}$ em 2000) e 6+3 (6 Mg ha ${ }^{-1}$ em 1993 e $3 \mathrm{Mg} \mathrm{ha}^{-1}$ em 2000).
Os resultados foram submetidos à análise de variância, e as médias foram comparadas pelo teste LSD, a 5\% de probabilidade. A análise de regressão foi usada para avaliar a relação de COT com $\mathrm{V}, \mathrm{m}$ e CTCe. O coeficiente de correlação de Pearson foi utilizado na avaliação do grau de afinidade entre COT e os atributos de fertilidade. Os coeficientes de correlação foram avaliados a 5 e $1 \%$ de probabilidade.

\section{Resultados e Discussão}

Após 15 anos da primeira aplicação e sete anos da reaplicação, a calagem promoveu variações nos atributos químicos do solo (Tabela 2). Quando comparado ao tratamento controle (sem aplicação de calcário), o tratamento $6+3$ promoveu aumento do $\mathrm{pH}$, do $\mathrm{Ca}^{2+}$ e do $\mathrm{Mg}^{2+}$ trocáveis, e diminuição do $\mathrm{H}+\mathrm{Al}^{3+}$ e o $\mathrm{Al}^{3+}$ trocável, até a camada de $10-20 \mathrm{~cm}$. Porém, as maiores modificações ocorreram nas camadas mais superficiais.

Os tratamentos $6+0$ e $0+3$ proporcionaram valores intermediários nos atributos químicos do solo. Observou-se diferença significativa quanto às variáveis $\mathrm{pH}, \mathrm{Ca}^{2+}$ e $\mathrm{Mg}^{2+}$ trocáveis, $\mathrm{H}+\mathrm{Al}^{3+}$ e $\mathrm{Al}^{3+}$ trocável apenas na camada de $0-2,5 \mathrm{~cm}$. Os atributos de fertilidade melhoraram com a aplicação de $6 \mathrm{Mg} \mathrm{ha}^{-1} \mathrm{de}$ calcário apenas em 1993. Essas melhorias nos atributos de fertilidade do solo são importantes para viabilizar maior produção das culturas. Segundo Caires et al. (2005), a calagem proporcionou, em 13 safras, ganho de $11 \%$ na produção acumulada de grãos. Além disso, o controle de $\mathrm{Al}^{3+}$ e o aumento de bases trocáveis podem promover aumento da produção de raízes (Caires et al., 2006b; Corrêa et al., 2008). Dessa forma, o aumento na produção de grãos, em solos que receberam calcário, resulta também em maior retorno de resíduos culturais no sistema (Tabela 1).

A calagem superficial também promoveu aumento no conteúdo de COT em todo o perfil do solo (Figura 1). Verificou-se, em comparação ao controle, maior aumento para o tratamento que recebeu calagem, tanto em 1993 quanto em 2000, com ganho até a última camada amostrada $(10-20 \mathrm{~cm})$.

As maiores diferenças nos teores de COT entre os tratamentos foram observadas na camada de $0-2,5 \mathrm{~cm}$. Nesta camada, o teor de COT no tratamento $6+3$ foi $29 \%$ superior ao $0+0$, e os teores diminuíram a partir da superfície - de 29,3 $\mathrm{g} \mathrm{kg}^{-1}$, na camada de 0-2,5 cm, para 
$16,9 \mathrm{~g} \mathrm{~kg}^{-1}$, na camada de $10-20 \mathrm{~cm}$, o que representa diminuição de $42 \%$. No tratamento controle, o teor de COT variou de $22,7 \mathrm{~g} \mathrm{~kg}^{-1}$, na camada superficial, para $15,4 \mathrm{~g} \mathrm{~kg}^{-1}$, na última camada amostrada, com decréscimo no perfil de $32 \%$. Os tratamentos $6+0$ e $0+3$ apresentaram gradiente intermediário de concentração do COT, com reduções da primeira para a última camada de 39 e $35 \%$, respectivamente.

O acúmulo de COT na camada superficial do solo, responsável por sua estratificação ao longo do perfil, é comumente observado em SPD e deve-se ao aporte contínuo de resíduos sem o revolvimento do solo (Franzluebbers, 2002; Sá \& Lal, 2009). A prática da calagem, ao promover melhorias nos atributos químicos do solo (Tabela 2) e maior produção das culturas (Caires et al., 2005, 2006b; Corrêa et al., 2008), eleva a quantidade de resíduos no sistema (Tabela 1). Portanto, em 15 anos de experimento, essa prática proporcionou acúmulo de COT, em especial nas camadas superficiais do solo (Figura 1).

Em estudo recente, Briedis et al. (2012) demonstraram que a prática da calagem em SPD, em longo período, promove acréscimo nos teores de
COT e em outros compartimentos da MOS, como polissacarídeos totais e lábeis e carbono extraído em água quente. Estes autores também observaram que o maior aumento proporcional de COT ocorre na fração lábil da MOS, a qual está amplamente relacionada aos resíduos culturais. Desse modo, a calagem, em longo período, promove maior retorno de $\mathrm{C}$ ao sistema via culturas, o que aumenta o COT no solo.

O conteúdo de NT também foi alterado pela calagem, tendo-se observado diferença significativa em todos os tratamentos, exceto na camada de 2,5-5 cm (Figura 1). Nas demais camadas, a calagem proporcionou aumento no NT, com maior ganho no tratamento $6+3$, na camada superficial de $0-2,5 \mathrm{~cm}$.

Além dos atributos de fertilidade, a calagem proporcionou aumento da saturação por bases (V) e promoveu decréscimo na saturação por $\mathrm{Al}^{3+}(\mathrm{m})$ do solo (Tabela 2). Estes dois fenômenos, em conjunto, foram os principais responsáveis pelo acúmulo de COT nos 15 anos de experimento (Figura 2). Esses resultados estão de acordo com os de estudo em solos ácidos da Austrália, no qual o aumento da fertilidade do solo, pela aplicação de $\mathrm{P}$ e calcário, em um

Tabela 2. Atributos químicos do solo, em diferentes camadas, afetados pela calagem superficial em um Latossolo Vermelho sob sistema plantio direto.

\begin{tabular}{|c|c|c|c|c|c|c|c|c|c|}
\hline \multirow[t]{2}{*}{$\overline{\operatorname{Dose}^{(1)}\left(\mathrm{Mg} \mathrm{ha}^{-1}\right)}$} & $\mathrm{pH} \mathrm{CaCl}{ }_{2}$ & $\mathrm{H}^{+}+\mathrm{Al}^{3+}$ & $\mathrm{Al}^{3+}$ & $\mathrm{Ca}^{2+}$ & $\mathrm{Mg}^{2+}$ & $\mathrm{K}^{+}$ & \multirow{2}{*}{$\begin{array}{l}\text { P Mehlich-1 } \\
\left(\mathrm{mg} \mathrm{dm}^{-3}\right)\end{array}$} & \multirow[t]{2}{*}{ Saturação por bases } & \multirow{2}{*}{ Saturação por $\mathrm{Al}^{3+}$} \\
\hline & \multirow{2}{*}{\multicolumn{8}{|c|}{ Camada de $0-2,5 \mathrm{~cm}$}} & \\
\hline & & & & & & & & & \\
\hline $0+0$ & 4,2 & 9,7 & 0,9 & 2,1 & 1,5 & 0,42 & 8,8 & 29,5 & 18,7 \\
\hline $6+0$ & 5,0 & 6,5 & 0,2 & 4,3 & 3,1 & 0,46 & 9,2 & 54,8 & 2,1 \\
\hline $0+3$ & 4,6 & 7,0 & 0,5 & 3,5 & 2,5 & 0,62 & 11,6 & 48,7 & 7,4 \\
\hline \multirow[t]{2}{*}{$6+3$} & 5,2 & 5,6 & 0,0 & 5,0 & 3,0 & 0,63 & 14,8 & 60,7 & 0,0 \\
\hline & \multicolumn{9}{|c|}{ Camada de $2,5-5,0 \mathrm{~cm}$} \\
\hline $0+0$ & 3,9 & 11,3 & 1,1 & 1,2 & 0,9 & 0,28 & 9,7 & 17,3 & 32,4 \\
\hline $6+0$ & 4,5 & 8,0 & 0,5 & 3,7 & 2,7 & 0,28 & 14,9 & 45,5 & 7,4 \\
\hline $0+3$ & 4,4 & 8,3 & 0,8 & 2,8 & 2,1 & 0,30 & 8,8 & 38,6 & 12,8 \\
\hline \multirow[t]{2}{*}{$6+3$} & 4,9 & 6,6 & 0,2 & 4,6 & 2,8 & 0,17 & 8,4 & 53,4 & 3,0 \\
\hline & \multicolumn{9}{|c|}{ Camada de $5-10 \mathrm{~cm}$} \\
\hline $0+0$ & 3,9 & 11,4 & 1,5 & 1,0 & 1,0 & 0,20 & 7,4 & 15,8 & 41,8 \\
\hline $6+0$ & 4,3 & 8,8 & 0,9 & 2,6 & 1,7 & 0,24 & 11,8 & 33,9 & 16,1 \\
\hline $0+3$ & 4,3 & 9,4 & 0,8 & 1,9 & 1,7 & 0,25 & 9,6 & 29,2 & 17,2 \\
\hline \multirow[t]{2}{*}{$6+3$} & 4,8 & 6,4 & 0,2 & 3,5 & 2,9 & 0,18 & 10,0 & 50,8 & 3,4 \\
\hline & \multicolumn{9}{|c|}{ Camada de $10-20 \mathrm{~cm}$} \\
\hline $0+0$ & 4,0 & 10,5 & 1,4 & 0,5 & 0,6 & 0,16 & 4,0 & 11,0 & 51,3 \\
\hline $6+0$ & 4,3 & 8,2 & 0,8 & 1,9 & 1,6 & 0,21 & 5,2 & 31,0 & 18,5 \\
\hline $0+3$ & 4,1 & 9,3 & 1,0 & 1,2 & 1,4 & 0,20 & 3,2 & 23,5 & 26,7 \\
\hline $6+3$ & 4,5 & 7,0 & 0,5 & 2,4 & 1,8 & 0,12 & 4,2 & 38,2 & 11,1 \\
\hline DMS & 0,3 & 1,3 & 0,3 & 1,0 & 0,7 & 0,21 & 7,2 & 10,3 & 8,7 \\
\hline
\end{tabular}

${ }^{(1)}$ Doses de calcário: 0+0 (0 Mg ha $\mathrm{em}^{-1} 1993$ e $\left.0 \mathrm{Mg} \mathrm{ha}^{-1} \mathrm{em}_{2000}\right)$, 6+0 (6 $\mathrm{Mg} \mathrm{ha}^{-1} \mathrm{em} 1993$ e $0 \mathrm{Mg} \mathrm{ha}^{-1} \mathrm{em} \mathrm{2000),0+3} \mathrm{(0} \mathrm{Mg} \mathrm{ha-1} \mathrm{em} 1993$ e 3 Mg ha- em 2000) e 6+3 (6 Mg ha-1 em 1993 e $3 \mathrm{Mg} \mathrm{ha}^{-1}$ em 2000). DMS, diferença mínima significativa, a 5\% de probabilidade. 
período de 68 anos, aumentou o estoque de COT em $11,8 \mathrm{Mg} \mathrm{ha}^{-1}$, na camada de 0-10 cm (Ridley et al., 1990). Em experimento de longa duração em solos com predominância de caulinita e ilita (Typic Haplustalf) na Índia, a calagem com a aplicação de fertilizantes (NPK) promoveu aumento significativo no COT após 29 anos, em comparação ao tratamento que recebeu somente NPK (Hati et al., 2008). O efeito da melhoria da fertilidade do solo no aumento de COT pode ser
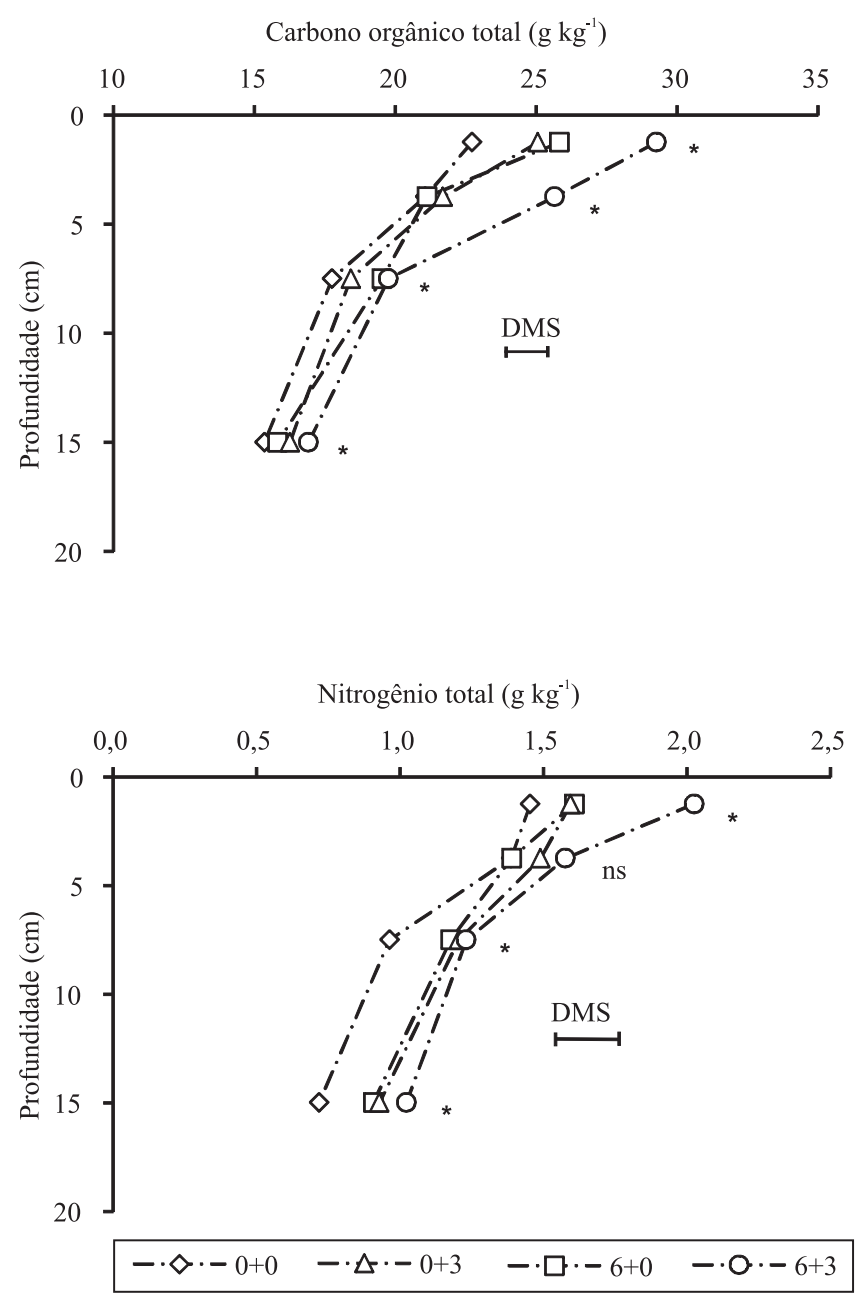

Figura 1. Conteúdo de carbono orgânico total (COT) e nitrogênio total (NT) no perfil de um Latossolo Vermelho sob sistema plantio direto, em função da aplicação de calcário na superfície: $0+0$ (0 Mg ha ${ }^{-1}$ em 1993 e $0 \mathrm{Mg} \mathrm{ha}^{-1}$ em 2000), $6+0\left(6 \mathrm{Mg} \mathrm{ha}^{-1} \mathrm{em} 1993\right.$ e $\left.0 \mathrm{Mg} \mathrm{ha}^{-1} \mathrm{em} 2000\right), 0+3\left(0 \mathrm{Mg} \mathrm{ha}^{-1}\right.$ em 1993 e $3 \mathrm{Mg} \mathrm{ha}^{-1}$ em 2000) e 6+3 (6 Mg ha- em 1993

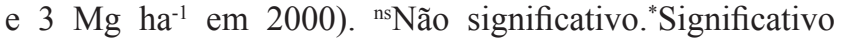
pelo teste diferença mínima significativa (DMS), a 5\% de probabilidade. atribuído, principalmente, ao aumento do retorno de resíduos nessas condições.

$\mathrm{O}$ aumento de COT, influenciado pela calagem, promoveu o aumento da CTCe no solo (Figura 3). Esses atributos apresentaram alta correlação $(p<0,01)$. $\mathrm{O}$ coeficiente angular da equação de regressão linear simples mostrou que a CTCe da matéria orgânica é de aproximadamente $215 \mathrm{cmol}_{\mathrm{c}} \mathrm{dm}^{-3}(0,37 \mathrm{x} 1.000 / 1,724)$. Soares \& Alleoni (2008), ao avaliar 30 diferentes tipos

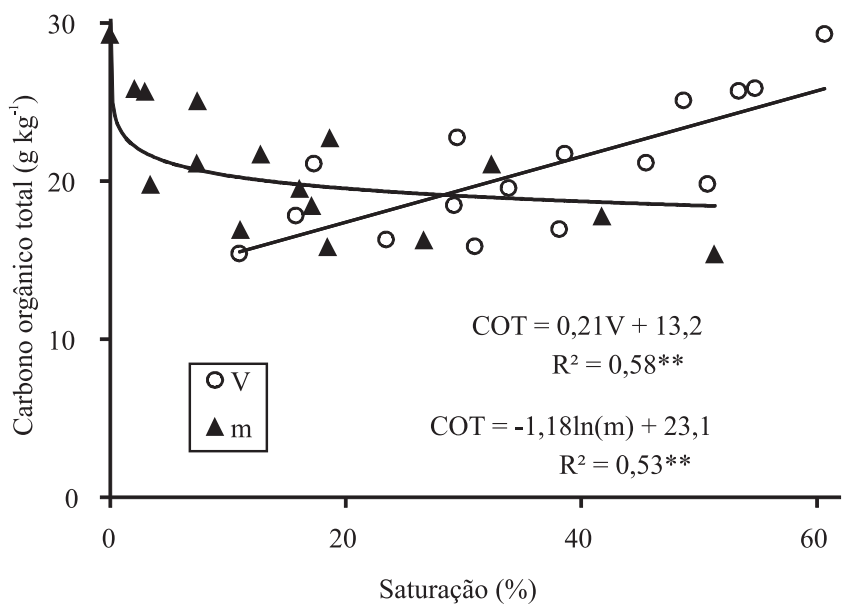

Figura 2. Relação da saturação por bases (V) e saturação por $\mathrm{Al}^{3+}(\mathrm{m})$ com o teor de carbono orgânico total (COT) no solo. ${ }^{* *}$ Significativo pelo teste diferença mínima significativa (DMS), a 1\% de probabilidade.

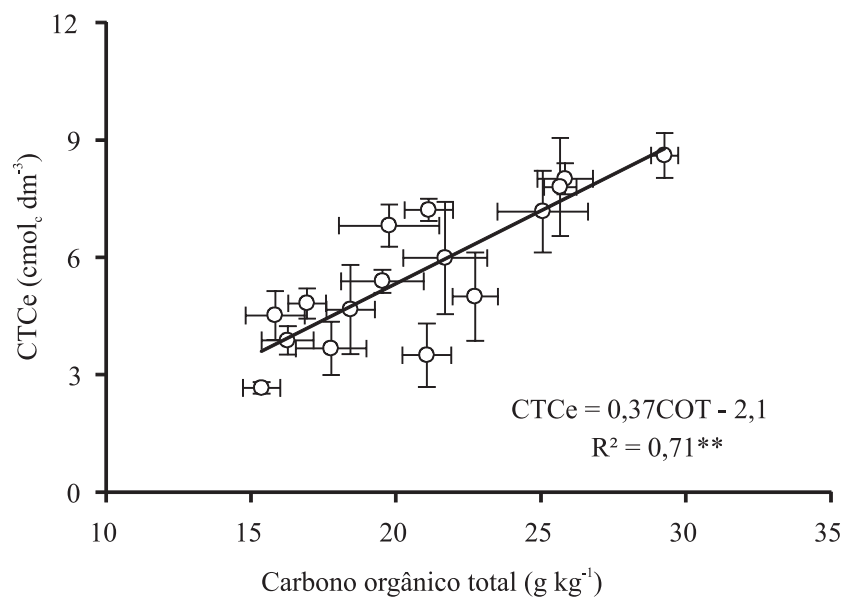

Figura 3. Relação entre capacidade de troca catiônica efetiva (CTCe) e teor de carbono orgânico total (COT) do solo. As barras representam o erro-padrão da média ( $\mathrm{n}=3$ ). ${ }^{* *}$ Significativo pelo teste diferença mínima significativa (DMS), a 1\% de probabilidade.

Pesq. agropec. bras., Brasília, v.47, n.7, p.1007-1014, jul. 2012 
de solos do Estado de São Paulo, obtiveram contribuição média do carbono na CTC de $164 \mathrm{cmol}_{\mathrm{c}} \mathrm{kg}^{-1}$, a qual foi 44 vezes superior à contribuição da fração argila (4 $\mathrm{cmol}_{\mathrm{c}} \mathrm{kg}^{-1}$ ). O aumento da CTC também foi relacionado ao aumento do COT em outros trabalhos realizados em ambientes subtropicais e tropicais (Sá et al., 2009; Anda \& Kurnia, 2010).

A CTC da matéria orgânica origina-se da dissociação de grupos funcionais, principalmente os carboxílicos, que são pH-dependentes (Sposito, 2008). Portanto, a prática da calagem promoveu aumento da CTCe tanto pelo aumento de carbono no perfil do solo (Figura 1) quanto pela elevação do pH (Tabela 2), que contribuiu para uma maior dissociação dos grupos funcionais da matéria orgânica acrescida. Nesse aspecto, a equação de regressão linear múltipla $(\mathrm{n}=48)$ do $\mathrm{pH}$ e do teor de COT (variáveis independentes) com a CTCe (variável dependente) do solo foi expressa por:

$$
\mathrm{CTCe}=-11,5+3,18 \mathrm{pH}+0,15 \mathrm{COT}\left(\mathrm{R}^{2}=0,92^{* * *}\right) .
$$

As correlações entre COT e os atributos de fertilidade do solo avaliados foram significativas (Tabela 3). A elevada correlação encontrada entre COT e Ca indica ter ocorrido a estabilização de COT nas argilas, por meio de ponte catiônica mediada pelo elemento (Bronick \& Lal, 2005). O aumento do $\mathrm{pH}$, promovido pela calagem, proporciona a dissociação de $\mathrm{H}^{+}$de grupos $\mathrm{OH}$ da matéria orgânica, da caulinita, e de óxidos de $\mathrm{Fe}$ e de $\mathrm{Al}$, o que acarreta aumento da CTCe (Alleoni et al., 2005). Aliado a isto, a maior disponibilidade de $\mathrm{Ca}^{2+}$ na solução do solo, também resultado da calagem, pode ter promovido a associação argilo-húmica.

Tabela 3. Matriz de correlação entre os atributos de fertilidade do solo.

\begin{tabular}{lcccccccc}
\hline & $\mathrm{COT}$ & $\mathrm{pH}$ & $\mathrm{Ca}$ & $\mathrm{Mg}$ & $\mathrm{K}$ & $\mathrm{P}$ & $\mathrm{CTCe}$ & $\mathrm{CTCp}$ \\
\hline $\mathrm{pH}$ & $0,66^{* *}$ & & & & & & & \\
$\mathrm{Ca}$ & $0,77^{* *}$ & $0,86^{* *}$ & & & & & & \\
$\mathrm{Mg}$ & $0,66^{* *}$ & $0,83^{* *}$ & $0,96^{* *}$ & & & & & \\
$\mathrm{~K}$ & $0,62^{* *}$ & $0,40^{* *}$ & $0,46^{* *}$ & $0,43^{* *}$ & & & & \\
$\mathrm{P}$ & $0,31^{*}$ & $0,28^{\text {ns }}$ & $0,28^{\text {ns }}$ & $0,20^{\text {ns }}$ & $0,32^{*}$ & & & \\
$\mathrm{CTCe}$ & $0,75^{* *}$ & $0,80^{* *}$ & $0,98^{* *}$ & $0,97^{* *}$ & $0,53^{* *}$ & $0,25^{\text {ns }}$ & & \\
$\mathrm{CTCp}$ & $0,68^{* *}$ & $0,30^{*}$ & $0,62^{* *}$ & $0,58^{* *}$ & $0,49^{* *}$ & $0,25^{\text {ns }}$ & $0,66^{* *}$ & \\
$\mathrm{SB}$ & $0,70^{* *}$ & $0,88^{* *}$ & $0,98^{* *}$ & $0,98^{* *}$ & $0,45^{* *}$ & $0,26^{\text {ns }}$ & $0,97^{* *}$ & $0,48^{* *}$ \\
\hline
\end{tabular}

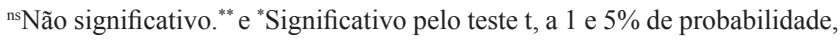
respectivamente. COT, carbono orgânico total; CTCe, capacidade de troca catiônica efetiva; CTCp, capacidade de troca catiônica potencial; SB, soma de bases.
A correlação existente de COT com P e K (Tabela 3) indica a possibilidade da matéria orgânica do solo disponibilizar esses elementos para as plantas. Em relação ao K, a sua correlação com o COT está relacionada à grande quantidade de resíduos culturais, pois aproximadamente $65-75 \%$ do $\mathrm{K}$ absorvido pelas plantas é proveniente de resíduos anteriores (Malavolta, 2006). Já a correlação entre COT e P pode ser atribuída ao aumento de cargas negativas com o aumento de COT, o que favorece a manutenção de P disponível (Negassa et al., 2008). O COT também pode aumentar a disponibilidade de $\mathrm{P}$, pois evita sua adsorção com outros elementos, como ferro e alumínio, com consequente conversão para formas indisponíveis (Santos \& Tomm, 2003).

A CTCp teve maior afinidade com o COT $(r=0,68)$, em comparação ao $\mathrm{pH}(\mathrm{r}=0,30)$ (Tabela 3). Portanto, o aumento da capacidade potencial de cargas negativas do solo foi mais influenciado pelo aumento de COT (Figura 2), em razão dos tratamentos com calagem, do que pelo aumento de $\mathrm{pH}$. Contudo, o $\mathrm{pH}$ apresentou correlação elevada com a CTCe $(r=0,80)$, em razão de seu aumento possibilitar a dissociação de grupamentos carboxílicos da MOS e grupamentos silanol e aluminol da caulinita (Alleoni et al., 2005). Assim, mudanças conjuntas ocorridas no $\mathrm{pH}$ e em COT, com a calagem, favoreceram o número de cargas negativas no solo (potenciais e efetivas). Isso é de suma importância em solos tropicais, cujos argilominerais dominantes são constituídos por caulinita e óxidos de ferro e alumínio.

\section{Conclusões}

1. A calagem superficial em sistema plantio direto, em longo período, proporciona aumento no conteúdo de carbono orgânico total (COT) e de $\mathrm{N}$ total.

2. O aumento de COT no solo está relacionado ao aumento de saturação por bases e à diminuição da saturação por alumínio.

3. O aumento de COT no solo proporciona elevação na capacidade de troca catiônica potencial e efetiva.

4. O COT no solo correlaciona-se aos conteúdos de $\mathrm{P}$ e $\mathrm{K}$.

\section{Agradecimentos}

À Fundação Araucária, pela concessão de bolsa; e à Fundação Agrisus - Agricultura Sustentável -, pelo apoio financeiro. 


\section{Referências}

ALLEONI, L.R.F.; IGLESIAS, C.S.M.; MELLO, S. de C.; CAMARGO, O.A. de; CASAGRANDE, J.C.; LAVORENTI, N.A. Atributos do solo relacionados à adsorção de cádmio e cobre em solos tropicais. Acta Scientiarum. Biological Sciences, v.27, p.729-737, 2005.

ANDA, M.; KURNIA, U. Restoring properties of artificially degraded ultisols and oxisols and the effect on crop yields under tropical conditions. Communications in Soil Science and Plant Analysis, v.41, p.553-570, 2010.

BORTOLUZZI, E.C.; RHEINHEIMER, D. dos S.; PETRY, C.; KAMINSKI, J. Contribuição de constituintes de solo à capacidade de troca de cátions obtida por diferentes métodos de extração. Revista Brasileira de Ciência do Solo, v.33, p.507-515, 2009.

BRIEDIS, C.; SÁ, J.C. de M.; CAIRES, E.F.; NAVARRO, J. de F.; INAGAKI, T.M.; BOER, A.; FERREIRA, A. de O.; QUADROS NETO, C.; CANALLI, L.B.; SANDOS, J.B. dos. Changes in organic matter pools and increases in carbon sequestration in response to surface liming in an oxisol under long-term no-till. Soil Science Society of America Journal, v.76, p.151-160, 2012.

BRONICK, C.J.; LAL, R. Soil structure and management: a review. Geoderma, v.124, p.3-22, 2005.

CAIRES, E.F.; ALLEONI, L.R.F.; CAMBRI, M.A.; BARTH, G. Surface application of lime for crop grain production under a no-till system. Agronomy Journal, v.97, p.791-798, 2005.

CAIRES, E.F.; BARTH, G.; GARBUIO, F.J. Lime application in the establishment of a no-till system for grain crop production in Southern Brazil. Soil and Tillage Research, v.89, p.3-12, $2006 \mathrm{a}$.

CAIRES, E.F.; CORRÊA, J.C.L.; CHURKA, S.; BARTH, G. Surface application of lime ameliorates subsoil acidity and improves root growth and yield of wheat in an acid soil under no-till system. Scientia Agricola, v.63, p.502-509, $2006 \mathrm{~b}$.

CAIRES, E.F.; GARBUIO, F.J.; CHURKA, S.; BARTH, G.; CORREAA, J.C.L. Effects of soil acidity amelioration by surface liming on no-till corn, soybean, and wheat root growth and yield. European Journal of Agronomy, v.28, p.57-64, 2008.

CLAESSEN, M.E.C. (Org.). Manual de métodos de análise de solo. 2.ed. rev. atual. Rio de Janeiro: Embrapa-CNPS, 1997. 212p. (Embrapa-CNPS. Documentos, 1).

CORRÊA, J.C.; BÜLL, L.T.; CRUSCIOL, C.A.C.; FERNANDES, D.M.; PERES, M.G.M. Aplicação superficial de diferentes fontes de corretivos no crescimento radicular e produtividade da aveia preta. Revista Brasileira de Ciência do Solo, v.32, p.1583-1590, 2008.

EKENLER, M.; TABATABAI, M.A. Effects of liming and tillage systems on microbial biomass and glycosidases in soils. Biology and Fertility of Soils, v.39, p.51-61, 2003.

ERNANI, P.R.; RIBEIRO, M.F.S.; BAYER, C. Chemical modifications caused by liming below the limed layer in a predominantly variable charge acid soil. Communications in Soil Science and Plant Analysis, v.35, p.889-901, 2004.
FONTANA, A.; PEREIRA, M.G.; LOSS, A.; CUNHA, T.J.F.; SALTON, J.C. Atributos de fertilidade e frações húmicas de um Latossolo Vermelho no Cerrado. Pesquisa Agropecuária Brasileira, v.41, p.847-853, 2006.

FRANZLUEBBERS, A.J. Soil organic matter stratification ratio as an indicator of soil quality. Soil and Tillage Research, v.66, p.95-106, 2002.

HATI, K.M.; SWARUP, A.; MISHRA, B.; MANNA, M.C.; WANJARI, R.H.; MANDAL, K.G.; MISRA, A.K. Impact of long-term application of fertilizer, manure and lime under intensive cropping on physical properties and organic carbon content of an Alfisol. Geoderma, v.148, p.173-179, 2008.

MALAVOLTA, E. Manual de nutrição mineral de plantas. Piracicaba: Ceres, 2006. 638p.

MIJANGOS, I.; ALBIZU, I.; EPELDE, L.; AMEZAGA, I.; MENDARTE, S.; GARBISU, C. Effects of liming on soil properties and plant performance of temperate mountainous grasslands. Journal of Environmental Management, v.91, p.2066-2074, 2010.

MOREIRA, A.; FAGERIA, N.K. Liming influence on soil chemical properties, nutritional status and yield of alfalfa grown in acid soil. Revista Brasileira de Ciência do Solo, v.34, p.1231-1239, 2010.

NEGASSA, W.; DULTZ, S.; SCHLICHTING, A.; LEINWEBER, P. Influence of specific organic compounds on phosphorus sorption and distribution in a tropical soil. Soil Science, v.173, p.587-601, 2008.

PAVAN, M.A.; BLOCH, M. de F.; ZEMPULSKI, H. da C.; MIYAZAWA, M.; ZOCOLER, D.C. Manual de análise química do solo e controle de qualidade. Londrina: Instituto Agronômico do Paraná, 1992. 38p. (IAPAR. Circular, 76).

RIDLEY, A.M.; SLATTERY, W.J.; HELYER, K.R.; COWLING, A. The importance of the carbon cycle to acidification of a grazed annual pasture. Australian Journal of Experimental Agriculture, v.30, p.529-537, 1990.

SÁ, J.C. de M.; CERRI, C.C.; LAL, R.; DICK, W.A.; PICCOLO, M. de C.; FEIGL, B.E. Soil organic carbon and fertility interactions affected by a tillage chornosequence in a Brazilian Oxisol. Soil and Tillage Research, v.104, p.56-64, 2009.

SÁ, J.C. de M.; CERRI, C.C.; LAL, R.; DICK, W.A.; VENZKE FILHO, S.P.; PICCOLO, M. de C.; FEIGL, B.E. Organic matter dynamics and sequestration rates for a tillage chronosequence in a Brazilian Oxisol. Soil Science Society of America Journal, v.65, p.1486-1499, 2001.

SÁ, J.C. de M.; LAL, R. Stratification ratio of soil organic matter pools as an indicator of carbon sequestration in a tillage chronosequence on a Brazilian Oxisol. Soil and Tillage Research, v.103, p.46-56, 2009.

SANTOS, H.G. dos; JACOMINE, P.K.T.; ANJOS, L.H.C. dos; OLIVEIRA, V.A. de; OLIVEIRA, J.B. de; COELHO, M.R.; LUMBRERAS, J.F.; CUNHA, T.J.F. (Ed.). Sistema brasileiro de classificação de solos. Rio de Janeiro: Embrapa Solos, 2006. $306 \mathrm{p}$. 
SANTOS, H.P.; TOMM, G.O. Disponibilidade de nutrientes e teor de matéria orgânica em função de sistemas de cultivo e de manejo de solo. Ciência Rural, v.33, p.477-486, 2003.

SOARES, M.R.; ALLEONI, L.R.F. Contribution of soil organic carbon to the ion exchange capacity of tropical soils. Journal of Sustainable Agriculture, v.32, p.439-462, 2008.

SOARES, M.R.; ALLEONI, L.R.F.; VIDAL-TORRADO, P.; COOPER, M. Mineralogy and ion exchange properties of the particle size fractions of some Brazilian soils in tropical humid areas. Geoderma, v.125, p.355-367, 2005.
SPOSITO, G. The chemistry of soils. $2^{\text {nd }}$ ed. New York: Oxford University, 2008. 330p.

WEBER, O.L. dos S.; CHITOLINA, J.C.; CAMARGO, O.A. de; ALLEONI, L.R.F. Structural and variable electric charges of highly weathered tropical soils. Revista Brasileira de Ciência do Solo, v.29, p.867-873, 2005.

YAO, H.Y.; BOWMAN, D.; RUFTY, T.; SHI, W. Interactions between $\mathrm{N}$ fertilization, grass clipping addition and $\mathrm{pH}$ in turf ecosystems: implications for soil enzyme activities and organic matter decomposition. Soil Biology and Biochemistry, v.41, p.1425-1432, 2009.

Recebido em 1o de fevereiro de 2012 e aprovado em 30 de abril de 2012 